\title{
Sucessão ecológica de um trecho de Floresta Ombrófila Densa de Terras Baixas, Carauari, Amazonas
}

\author{
Rosival Barros de Andrade Lima1, José Antônio Aleixo da Silva¹, Luiz Carlos Marangon¹, Rinaldo Luiz Caraciolo Ferreira1, \\ Roseane Karla Soares da Silva ${ }^{1}$
}

1 Universidade Federal Rural de Pernambuco (UFRPE), Rua Dom Manoel de Medeiros, s/n, Dois Irmãos, CEP 52171-900, Recife, PE, Brasil

"Autor correspondente:

rosival_barros@yahoo.com.br

Termos para indexação:

Amazônia

Florística

Grupos ecológicos

Index terms:

Amazon

Floristic

Ecological groups

\section{Histórico do artigo:}

Recebido em 29 mar 2011

Aprovado em 05 set 2011

Publicado em 30 set 2011

doi: 10.4336/2011.pfb.31.67.161
Resumo - O presente trabalho foi realizado em um trecho de Floresta Ombrófila Densa de Terras Baixas no Município de Carauari, Amazonas, tendo como objetivo estimar a composição florística do estrato arbóreo e classificar as espécies nos respectivos grupos ecológicos, a fim de se obter informações sobre a atual situação do fragmento florestal, para embasar estratégias de conservação e preservação, bem como embasar a formulação de pesquisas que visem à disseminação do conhecimento e sua aplicação na produção sustentável. O inventário florestal foi realizado em uma área de 275 ha, cortada por três transectos $(822 \mathrm{~m}, 1.265 \mathrm{~m}$ e $2.349 \mathrm{~m})$, totalizando $4.436 \mathrm{~m}$. Foram instaladas parcelas de $20 \mathrm{~m}$ x $25 \mathrm{~m}$, equidistantes a cada $50 \mathrm{~m}$ e intercaladas à direita e à esquerda da linha do transecto, totalizando 66 unidades amostrais. Foram identificados e medidos todos os indivíduos arbóreos que apresentaram circunferência a 1,30 m do solo (CAP) $\geq 25 \mathrm{~cm}$. Registraram-se 3.050 indivíduos, distribuídos em 133 espécies, 93 gêneros e 49 famílias. Quanto à classificação sucessional, as espécies de início de sucessão (pioneiras + secundárias iniciais) foram em maior número, demonstrando características de uma floresta em estágio inicial no desenvolvimento sucessional.

\section{Ecological succession of a stretch of Dense Rain Forest in the Lowlands, Carauari, Amazonas, Brazil}

\begin{abstract}
This work was carried out in a stretch of Dense Rain Forest in the lowland county of Carauari, Amazonas, aiming to estimate the species composition of tree layer and classify the species in their ecological groups in order to obtain information about the current situation of the forest fragment, to be the basis for strategies for conservation and preservation as well as the basis for formulating research aimed at the dissemination of knowledge and its application to sustainable production. The forest inventory was conducted in an area of $275 \mathrm{ha}$, crossed by three transects $(822$ $\mathrm{m}, 1,265 \mathrm{~m}$ and $2,349 \mathrm{~m}$ ), totaling $4.436 \mathrm{~m}$. Plots were installed in $20 \mathrm{~m} \times 25 \mathrm{~m}, 50$ $\mathrm{m}$ equidistant, merged to the right and left of the line transect, totaling 66 sampling units. All tree individuals trees that had a circumference of $1,30 \mathrm{~m}$ above the ground $(\mathrm{CAP}) \geq 25 \mathrm{~cm}$ were identified and measured. There were 3,050 individuals distributed in 133 species, 93 genera and 49 families. It was observed that the species of early succession (pioneer + early secondary) were more numerous, showing characteristics of a forest in early successional stage.
\end{abstract}




\section{Introdução}

O desconhecimento de padrões ecológicos aliado a sistemáticas intervenções em florestas tropicais, especialmente na Amazônia brasileira, tem gerado grandes impactos ambientais. As suas consequências, em algumas regiões, requerem ações conservacionistas imediatas (Pinheiro et al., 2007).

Entre as informações ecológicas relevantes para um manejo adequado, estão os grupos ecológicos em que se distribuem as espécies florestais (Swaine \& Whitmore, 1988). Para Rodrigues et al. (1998) um dos subsídios importantes para a conservação e recuperação das formações florestais parte da análise do estágio sucessional desses fragmentos. A separação das espécies arbóreas em grupos ecológicos é uma maneira de manejar o grande número de espécies da floresta tropical, mediante seu agrupamento por funções semelhantes e de acordo com as suas exigências (Rocha et al., 2008).

De acordo com Maciel et al. (2002), a luz é fundamental como fonte essencial e direta de energia para o desenvolvimento de todos os vegetais, e a importância desse fator tem levado diversos autores a classificar as espécies florestais em grupos ecológicos distintos, de acordo com sua capacidade de adaptação às condições de luminosidade ambiental, cujo conhecimento é chave importante para a compreensão da dinâmica da floresta e seu manejo. Porém, conforme Paula et al. (2004), a grande plasticidade apresentada pelas espécies dificulta a determinação dos critérios de classificação.

Para Ferraz et al. (2004), o conhecimento gerado pelos estudos dos grupos sucessionais pode ser aplicado diretamente na conservação, no manejo sustentado da floresta e na reabilitação de áreas degradadas. Além disso, diante do grande número de espécies madeireiras da Amazônia, o agrupamento em características ecológicas e silviculturais semelhantes facilitará a definição de metodologias e práticas aplicáveis ao manejo, principalmente das espécies que podem ser exploradas comercialmente.

Outra informação utilizada para a compreensão da sucessão é a distribuição diamétrica. De acordo com Paula et al. (2004), a distribuição diamétrica tem sido utilizada frequentemente em trabalhos de manejo florestal aplicado a povoamentos inequiâneos.

A compreensão da sucessão ecológica de um fragmento de floresta tropical é importante para tentar entender como as comunidades biológicas estão se regenerando e sobrevivendo em uma paisagem cada vez mais fragmentada. Dessa forma, esse estudo teve como objetivo caracterizar o estágio sucessional de um trecho de Floresta Ombrófila Densa de Terras Baixas, em uma Reserva de Desenvolvimento Sustentável (RDS) no Município de Carauari, sudoeste do Estado do Amazonas, com intuito de subsidiar futuros programas de restauração e de conservação dos recursos florestais.

\section{Material e métodos}

\section{Localização e caracterização da área de estudo}

O presente trabalho foi desenvolvido em um trecho de Floresta Ombrófila Densa de Terras Baixas (IBGE, 1992), na comunidade do Pupunha (67\%46’37.4" W e $05^{\circ} 35^{\prime} 45.2^{\prime}$ S, $93 \mathrm{~m}$ de altitude), pertencente à Reserva de Desenvolvimento Sustentável Uacari, situada no Município de Carauari, sudoeste do Estado do Amazonas, cuja área é de 632.949 ha.

A região da RDS Uacari não foi uma exceção na história em relação à exploração madeireira. Segundo relatos, quase a totalidade de sua área de fácil acesso, principalmente a várzea, foi explorada ao longo das últimas décadas. Por uma questão de mercado e logística de transporte, a variedade de espécies exploradas era pequena, pois foram priorizadas as espécies madeireiras que flutuavam (Andretti et al., 2006).

Com a criação da reserva, a comercialização de madeira foi proibida até que fosse elaborado o plano de uso, documento que regulariza tal atividade. Atualmente, é uma atividade que se restringe a atender as demandas de subsistência das famílias da reserva. Os principais usos são a construção de casas e a fabricação de canoas (Andretti et al., 2006).

O clima dessa região, segundo a classificação de Köppen é Am - Tropical Chuvoso (Vianello \& Alves, 2000), com precipitação média anual de $2.500 \mathrm{~mm}$. A temperatura média do ar é de $24{ }^{\circ} \mathrm{C}$, com pequena amplitude térmica e a umidade geralmente permanece acima de 90\%. Os meses mais chuvosos vão de novembro a abril (Ibama, 2011).

Os solos são classificados como Argissolo Vermelho amarelo Álico de argila de atividade baixa, entrecortadas pela Lateritas Hidromórficas Álicas. Ocorrem também pequenas manchas de Latossolo Vermelho amarelo Álico (Brasil, 1977; Santos et al., 2006). 


\section{Amostragem}

Foi realizado levantamento sistemático numa área de 275 hectares, por meio de dois transectos no sentido Norte/Sul, com $822 \mathrm{~m}$ e $1.265 \mathrm{~m}$ de comprimento, respectivamente, e um no sentido Leste/Oeste com 2.349 m de comprimento, todos alinhados com auxílio de uma bússola. Ao longo dos transectos, foram instaladas parcelas de $20 \mathrm{~m} \times 25 \mathrm{~m}$, equidistantes a cada $50 \mathrm{~m}$ e intercaladas à direita e à esquerda da linha do transecto, totalizando 66 unidades amostrais, o equivalente a 3,3 hectares de área amostrada (Figura 1).

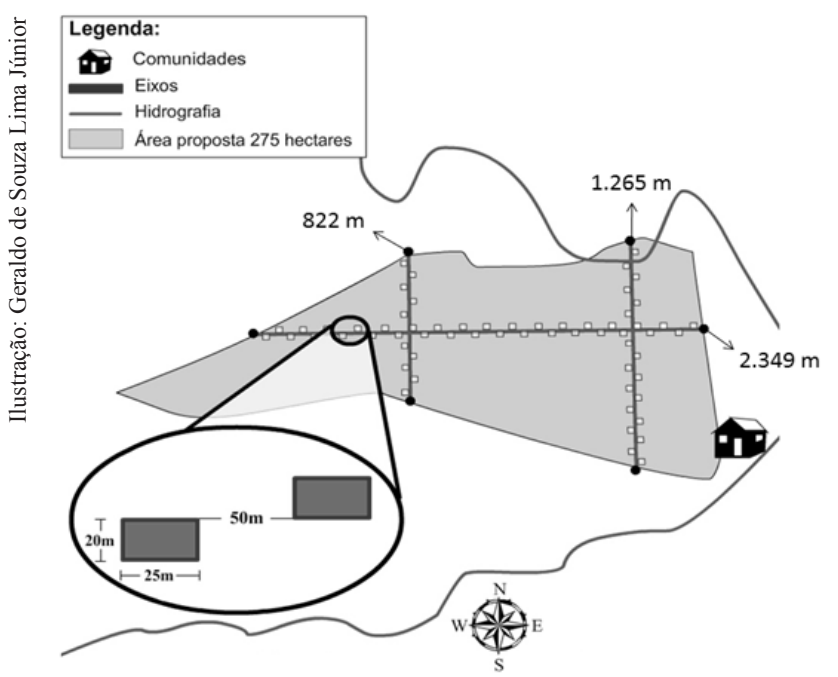

Figura 1. Croqui da área de estudo, localizada na comunidade do Pupunha, RDS Uacari, Carauari, AM.

Todas as unidades amostrais foram georreferenciadas e todos os indivíduos arbóreos que apresentaram circunferência à altura do peito (CAP) (a 1,30 m do nível do solo) $\geq 25 \mathrm{~cm}$ foram mensurados. Os indivíduos amostrados receberam placas de PVC com numeração crescente, as quais foram fixadas com prego.

O material botânico coletado foi encaminhado ao herbário da Universidade Federal do Amazonas (UFAM), para identificação botânica. As determinações foram feitas por meio de comparações com exsicatas já determinadas e depositadas no herbário, consulta a especialistas e à literatura especializada. A identificação taxonômica ocorreu de acordo com o sistema de classificação de Cronquist (1988).

\section{Análise de dados}

A suficiência amostral foi determinada considerando os estimadores da amostragem inteiramente aleatória (Meunier et al., 2002 e Soares et al., 2007). Utilizando o número de árvores amostradas por parcela, calculou-se o tamanho da amostra por meio da seguinte expressão:

em que $n=\frac{t_{\alpha / 2}^{2} \cdot(C V)^{2}}{(E \%)^{2}}$

$\mathrm{t}_{\alpha / 2}=$ valor tabelado da distribuição $\mathrm{t}$ de Student $(\alpha$ $5 \%, \mathrm{n}-1 \mathrm{gl})$;

$\mathrm{CV}=$ coeficiente de variação, em percentagem;

$\mathrm{E} \%=$ erro de amostragem.

Foi admitido um erro amostral (E\%) de 10\% e o nível de probabilidade de $95 \%$ usando a seguinte fórmula:

em que $E \%= \pm \frac{S_{\bar{Y}} \cdot t_{\propto / 2}}{\bar{Y}}$

$S_{\bar{Y}}=$ erro-padrão da média;

$\mathrm{t}_{\alpha / 2}=$ valor tabelado da distribuição $\mathrm{t}$ de Student $(\alpha$ $5 \%, \mathrm{n}-1 \mathrm{gl})$

$\mathrm{Y}=$ média do número de árvores por parcela.

As espécies levantadas foram classificadas de acordo com a proposta de Gandolfi et al. (1995), que as distinguem como pioneiras, secundárias iniciais, secundárias tardias e sem caracterização, em que: Pioneiras (P) - espécies claramente dependentes de luz que não ocorrem no sub-bosque, desenvolvendo-se em clareiras ou nas bordas da floresta; Secundárias iniciais (SI) - espécies que ocorrem em condições de sombreamento médio ou luminosidade não muito intensa, ocorrendo em clareiras pequenas, bordas de clareiras grandes, bordas da floresta ou no sub-bosque não densamente sombreado; Secundárias tardias (ST) - espécies que se desenvolvem no sub-bosque em condições de sombra leve ou densa, podendo aí permanecer toda a vida ou então crescer até alcançar o dossel ou a condição de emergente; Sem caracterização (SC) - espécies que em função da carência de informações não puderam ser incluídas em nenhuma das categorias anteriores.

Para a classificação das espécies encontradas no presente trabalho também foram reunidas informações dos estudos realizados por Gama et al. (2002), Silva et al. (2003), Santos et al. (2004), Carvalho et al. (2007), Marangon et al. (2007), Souza (2008), Amaral et al. (2009), Figueiredo et al. (2009) e Prado Júnior et al. (2010), além das observações realizadas em campo.

Para cada espécie, foram calculados os seguintes parâmetros quantitativos (em sua forma relativa): densidade, dominância, frequência e valor de importância (VI) (Müeller-Dombois \& Ellemberg, 1974). Além da 
análise dos grupos sucessionais das espécies amostradas, para identificação do estágio de desenvolvimento da floresta, analisou-se também a proporção entre o número de indivíduos, a frequência e a dominância dos diferentes grupos ecológicos (Prado Júnior et al., 2010).

Segundo Soares et al. (2007), pelo agrupamento dos diâmetros das árvores em classes, pode-se caracterizar a distribuição diamétrica de uma floresta. No Brasil, a maioria dos trabalhos utiliza amplitudes de $2 \mathrm{~cm}$ a $2,5 \mathrm{~cm}$ para plantios, e entre $5 \mathrm{~cm}$ e $10 \mathrm{~cm}$ para florestas inequiâneas (naturais). Sendo assim, para a análise da distribuição diamétrica, foi gerado um gráfico com o número de indivíduos por classe de diâmetro, no qual as classes diamétricas têm amplitudes de $10 \mathrm{~cm}$, sendo o início da primeira classe $7,95 \mathrm{~cm}$ (correspondente ao valor mínimo de diâmetro adotado como critério de inclusão no levantamento), e o primeiro centro de classe, $12,95 \mathrm{~cm}$.

\section{Resultados e discussão}

Foram amostrados 3.050 indivíduos de porte arbóreo, distribuídos em 133 espécies, pertencentes a 49 famílias botânicas e 93 gêneros. Dessas, 31 (23,31\%) foram identificadas em nível de gênero, 95 (71,43\%) em nível de espécie e sete $(5,26 \%)$ permanecem indeterminadas (Tabela 1).

Tabela 1. Florística e classificação sucessional das espécies arbóreas amostradas em uma área de Floresta Ombrófila Densa de Terras Baixas, na RDS Uacari, Carauari, AM, em ordem alfabética de família, gênero e espécies.

\begin{tabular}{|c|c|c|c|c|}
\hline Família/espécie & Nome vulgar & $\mathbf{N i}$ & DA & GE \\
\hline \multicolumn{5}{|l|}{ Anacardiaceae } \\
\hline Anacardium giganteum W. Hancock ex Engl. & Caju-bravo & 4 & 1,21 & $\mathrm{Si}$ \\
\hline Anacardium spruceanum Benth. ex Engl. & Cajuí & 16 & 4,85 & $\mathrm{P}$ \\
\hline \multicolumn{5}{|l|}{ Annonaceae } \\
\hline Guatteria poeppigiana Mart. & Embira-preta & 1 & 0,30 & $\mathrm{Si}$ \\
\hline Xylopia benthamii R. E. Fr. & Embireira-amarela & 1 & 0,30 & $\mathrm{Si}$ \\
\hline Xylopia nitida Dunal & Embireira & 177 & 53,64 & $\mathrm{Si}$ \\
\hline \multicolumn{5}{|l|}{ Apocynaceae } \\
\hline Aspidosperma excelsum Benth. & Carapanaúba & 5 & 1,52 & St \\
\hline Couma guianensis Aubl & Sorva & 13 & 3,94 & $\mathrm{St}$ \\
\hline Himatanthus sucuuba (Spruce ex Müll. Arg.) Woodson & Sucuúba & 5 & 1,52 & $\mathrm{P}$ \\
\hline \multicolumn{5}{|l|}{ Bignoniaceae } \\
\hline Tabebuia serratifolia (Vahl) G. Nicholson. & Ipê & 1 & 0,30 & $\mathrm{St}$ \\
\hline \multicolumn{5}{|l|}{ Bombacaceae } \\
\hline Bombax munguba Mart. \& Zucc. & Munguba-da-terra-firme & 1 & 0,30 & $P$ \\
\hline Pachira aquatica Aubl. & Mungubarana & 5 & 1,52 & $\mathrm{P}$ \\
\hline \multicolumn{5}{|l|}{ Boraginaceae } \\
\hline Cordia sp. & Grão-de-galo & 1 & 0,30 & $\mathrm{Si}$ \\
\hline \multicolumn{5}{|l|}{ Burseraceae } \\
\hline Protium heptaphyllum (Aubl.) March. & Amescla-de-cheiro & 115 & 34,85 & $\mathrm{Si}$ \\
\hline Protium trifoliolatum Engl. & Breu-branco & 44 & 13,33 & $\mathrm{Si}$ \\
\hline
\end{tabular}


Tabela 1. Continuação.

\begin{tabular}{|c|c|c|c|c|}
\hline Família/espécie & Nome vulgar & $\mathbf{N i}$ & DA & GE \\
\hline \multicolumn{5}{|l|}{ Caesalpiniaceae } \\
\hline Apuleia molaris Spruce ex Benth. & Garapeira & 22 & 6,67 & St \\
\hline Campsiandra comosa Benth. & Capurana & 18 & 5,45 & $\mathrm{Sc}$ \\
\hline Cassia leiandra Benth. & Marimari & 3 & 0,91 & $\mathrm{Si}$ \\
\hline Copaifera guyanensis Desf. & Copaíba & 1 & 0,30 & St \\
\hline Copaifera multijuga Hayne & Copaíba & 8 & 2,42 & St \\
\hline Copaifera piresii Ducke. & Copaíba & 3 & 0,91 & St \\
\hline Copaifera $\mathrm{sp}$ & Copaíba & 6 & 1,82 & St \\
\hline Hymenaea sp. 1 & Farinheira & 2 & 0,61 & St \\
\hline Hymenaea sp. 2 & Jatobarana & 1 & 0,30 & $\mathrm{St}$ \\
\hline Macrolobium acacifolium (Benth.) Benth. & Arapari & 1 & 0,30 & St \\
\hline Mora paraensis (Ducke) Ducke & Pracuúba & 3 & 0,91 & $\mathrm{Si}$ \\
\hline Peltogyne catingae Ducke & Violeta & 5 & 1,52 & $\mathrm{St}$ \\
\hline Peltogyne paniculata Benth. & Roxinho & 12 & 3,64 & St \\
\hline Tachigali paniculata Aubl. & Taxi & 24 & 7,27 & $\mathrm{St}$ \\
\hline \multicolumn{5}{|l|}{ Capparaceae } \\
\hline Crateva sp. & Trapiá & 7 & 2,12 & $\mathrm{Sc}$ \\
\hline \multicolumn{5}{|l|}{ Caryocaraceae } \\
\hline Caryocar glabrum Pers. & Piquiarana & 1 & 0,30 & St \\
\hline \multicolumn{5}{|l|}{ Cecropiaceae } \\
\hline Cecropia sp. & Imbaúba & 7 & 2,12 & $\mathrm{P}$ \\
\hline Pourouma sp. & Torém & 56 & 16,97 & $\mathrm{P}$ \\
\hline \multicolumn{5}{|l|}{ Celastraceae } \\
\hline Goupia glabra Aubl. & Cupiúba & 8 & 2,42 & $\mathrm{Si}$ \\
\hline \multicolumn{5}{|l|}{ Chrysobalanaceae } \\
\hline Couepia guianensis Aubl. & Castanha-de-cutia & 2 & 0,61 & $\mathrm{St}$ \\
\hline Licania apetala (E. Mey.) Fritsch & Farinha-seca & 19 & 5,76 & $\mathrm{Si}$ \\
\hline Licania canescens Benoist & Caraipé & 72 & 21,82 & $\mathrm{Si}$ \\
\hline Licania oblongifolia Standl. & Macucu & 202 & 61,21 & $\mathrm{Si}$ \\
\hline \multicolumn{5}{|l|}{ Clusiaceae } \\
\hline Calophyllum brasiliense Cambess. & Jacareúba & 1 & 0,30 & $\mathrm{St}$ \\
\hline Rheedia sp. & Bacuri & 65 & 19,70 & $\mathrm{Si}$ \\
\hline Tovomita caloneura A. C. Sm. & Pachubarana & 28 & 8,48 & $\mathrm{Si}$ \\
\hline Vismia guianensis (Aubl.) Pers. & Lacre & 7 & 2,12 & $\mathrm{P}$ \\
\hline \multicolumn{5}{|l|}{ Cochlospermaceae } \\
\hline Cochlospermum orinoccense (H. B. K.) Steud. & Algodão-bravo & 1 & 0,30 & $\mathrm{P}$ \\
\hline \multicolumn{5}{|l|}{ Combretaceae } \\
\hline Buchenavia grandis Ducke & Tanibuca & 19 & 5,76 & $\mathrm{Si}$ \\
\hline \multicolumn{5}{|l|}{ Elaeocarpaceae } \\
\hline Sloanea excelsa Ducke & Urucurana & 5 & 1,52 & $\mathrm{Si}$ \\
\hline \multicolumn{5}{|l|}{ Euphorbiaceae } \\
\hline Conceveiba martiana Baill. & Algodão & 4 & 1,21 & St \\
\hline Hevea brasiliensis (Willd. ex A. Juss) Müll. Arg. & Seringueira & 38 & 11,52 & St \\
\hline Mabea angustifolia Spruce ex Benth & Taquari & 15 & 4,55 & $\mathrm{P}$ \\
\hline Piranhea trifoliolata var. pubescens Radcl.-Sm. & Piranheiro & 1 & 0,30 & $\mathrm{Si}$ \\
\hline Pogonophora sp. & Amarelinho & 1 & 0,30 & $\mathrm{P}$ \\
\hline \multicolumn{5}{|l|}{ Fabaceae } \\
\hline Dipteryx odorata (Aubl.) Willd. & Cumaru & 12 & 3,64 & St \\
\hline Erythrina fusca Lour. & Mulungu & 17 & 5,15 & $\mathrm{P}$ \\
\hline Ormosia sp. & Cernambi-de-macaco & 41 & 12,42 & $\mathrm{P}$ \\
\hline Swartzia acuminata Willd. ex Vogel & Pitaíca & 2 & 0,61 & $\mathrm{Si}$ \\
\hline Swartzia laevicarpa Amshoff & Saboeira & 1 & 0,30 & St \\
\hline Swartzia sp. & Coração-de-negro & 8 & 2,42 & $\mathrm{St}$ \\
\hline
\end{tabular}


Tabela 1. Continuação.

\begin{tabular}{|c|c|c|c|c|}
\hline Família/espécie & Nome vulgar & $\mathbf{N i}$ & DA & GE \\
\hline \multicolumn{5}{|l|}{ Flacourtiaceae } \\
\hline Casearia grandiflora Cambess. & Sardinheira & 6 & 1,82 & $\mathrm{Si}$ \\
\hline \multicolumn{5}{|l|}{ Humiriaceae } \\
\hline Sacoglottis guianensis Benth & Uxirana & 7 & 2,12 & $\mathrm{St}$ \\
\hline Vantanea macrocarpa Ducke & Quebra-machado & 1 & 0,30 & $\mathrm{Si}$ \\
\hline Vantanea sp. & Uxirana & 9 & 2,73 & St \\
\hline \multicolumn{5}{|l|}{ Lauraceae } \\
\hline Aniba hostmanniana (Nees) Mez & Louro-amarelo & 24 & 7,27 & St \\
\hline Licaria cannella (Meisn.) Kosterm. & Louro-preto & 38 & 11,52 & St \\
\hline Licaria puchury-major (Mart.) Kosterm. & Louro-puxuri & 21 & 6,36 & St \\
\hline Licaria sp. & Louro-chumbo & 39 & 11,82 & St \\
\hline Mezilaurus itauba (Meisn.) Taub. ex Mez & Louro-itaúba & 2 & 0,61 & $\mathrm{St}$ \\
\hline Ocotea cymbarum Kunth & Louro-mamuri & 21 & 6,36 & St \\
\hline Ocotea $\mathrm{sp} .1$ & Louro-arabá & 2 & 0,61 & St \\
\hline Ocotea sp. 2 & Louro-babão & 22 & 6,67 & $\mathrm{St}$ \\
\hline Ocotea sp. 3 & Louro-bosta & 12 & 3,64 & St \\
\hline \multicolumn{5}{|l|}{ Lecythidaceae } \\
\hline Bertholletia excelsa Bonpl. & Castanheira & 2 & 0,61 & St \\
\hline Couratari oblongifolia Ducke \& R. Knuth & Currimboque & 12 & 3,64 & $\mathrm{St}$ \\
\hline Eschweilera odora (Poepp. ex O. Berg) Miers & Matamatá & 239 & 72,42 & $\mathrm{Si}$ \\
\hline Lecythis $\mathrm{sp} .1$ & Ripeira & 24 & 7,27 & St \\
\hline Lecythis sp. 2 & Castanharana & 6 & 1,82 & $\mathrm{St}$ \\
\hline \multicolumn{5}{|l|}{ Malpighiaceae } \\
\hline Byrsonima sp. & Murici & 1 & 0,30 & St \\
\hline \multicolumn{5}{|l|}{ Melastomataceae } \\
\hline Bellucia dichotoma Cogn. & Papaterra & 4 & 1,21 & $\mathrm{Si}$ \\
\hline Bellucia grossularioides (L.) Triana & Araçá-de-anta & 1 & 0,30 & $\mathrm{Si}$ \\
\hline Bellucia sp. & Umbigo-de-anta & 2 & 0,61 & $\mathrm{Si}$ \\
\hline \multicolumn{5}{|l|}{ Meliaceae } \\
\hline Carapa guianensis Aubl. & Andiroba & 2 & 0,61 & St \\
\hline Cedrela odorata $\mathrm{L}$. & Cedro & 1 & 0,30 & $\mathrm{Si}$ \\
\hline Trichilia micrantha Benth. & Jitó & 5 & 1,52 & St \\
\hline \multicolumn{5}{|l|}{ Mimosaceae } \\
\hline Abarema sp. & Cor-de-munis & 29 & 8,79 & $\mathrm{P}$ \\
\hline Dinizia excelsa Ducke & Angelim-pedra & 1 & 0,30 & St \\
\hline Inga capitata Desv. & Ingá-ferro & 1 & 0,30 & $\mathrm{Si}$ \\
\hline Inga sp. & Ingazeira & 219 & 66,36 & $\mathrm{Si}$ \\
\hline Parkia nitida Miq. & Faveira & 8 & 2,42 & $\mathrm{Si}$ \\
\hline \multicolumn{5}{|l|}{ Moraceae } \\
\hline Brosimopsis oblongifolia Ducke & Manichi & 5 & 1,52 & $\mathrm{Sc}$ \\
\hline Brosimum guianense (Aubl.) Huber & Amapá-amargo & 5 & 1,52 & $\mathrm{Si}$ \\
\hline Brosimum paraense Huber & Muirapiranga & 13 & 3,94 & $\mathrm{Si}$ \\
\hline Brosimum utile (Kunth) Oken ex J. Presl & Garrote & 17 & 5,15 & $\mathrm{Si}$ \\
\hline Castilla ulei Warb. & Caucho & 7 & 2,12 & $\mathrm{Sc}$ \\
\hline Clarisia racemosa Ruiz \& Pav. & Guariúba & 3 & 0,91 & $\mathrm{P}$ \\
\hline Ficus anthelmintica Mart. & Caxinguba & 1 & 0,30 & St \\
\hline Ficus maxima Mill. & Gameleira & 6 & 1,82 & St \\
\hline Ficus sp. & Apuí & 18 & 5,45 & St \\
\hline Maquira guianensis Aubl. & Muiratinga & 201 & 60,91 & $\mathrm{Si}$ \\
\hline \multicolumn{5}{|l|}{ Myristicaceae } \\
\hline Iryanthera tricornis Ducke & Punã & 65 & 19,70 & St \\
\hline Osteophloeum platyspermum (Spruce ex A. DC.) Warb. & Ucuubão & 5 & 1,52 & St \\
\hline Virola multiflora (Stand1.) A. C. Sm. & Virola & 121 & 36,67 & St \\
\hline Virola venosa (Benth.) Warb. & Ucuúba & 16 & 4,85 & St \\
\hline
\end{tabular}


Tabela 1. Continuação.

\begin{tabular}{|c|c|c|c|c|}
\hline Família/espécie & Nome vulgar & $\mathbf{N i}$ & DA & GE \\
\hline $\begin{array}{l}\text { Myrtaceae } \\
\quad \text { Eugenia paraensis } \mathrm{O} \text {. Berg } \\
\quad \text { Eugenia patrisii } \text { Vahl }\end{array}$ & $\begin{array}{l}\text { Araçá-da-mata } \\
\text { Araçarana }\end{array}$ & $\begin{array}{r}172 \\
15\end{array}$ & $\begin{array}{c}52,12 \\
4,55\end{array}$ & $\begin{array}{l}\mathrm{Si} \\
\mathrm{St}\end{array}$ \\
\hline $\begin{array}{l}\text { Nyctaginaceae } \\
\quad \text { Neea oppositifolia Ruiz \& Pav. }\end{array}$ & João-mole & 52 & 15,76 & $\mathrm{P}$ \\
\hline $\begin{array}{l}\text { Olacaceae } \\
\qquad \text { Minquartia guianensis Aubl. }\end{array}$ & Aquariquara & 1 & 0,30 & $\mathrm{Si}$ \\
\hline $\begin{array}{l}\text { Polygonaceae } \\
\qquad \text { Coccoloba } \mathrm{sp} .\end{array}$ & Santa-maria & 5 & 1,52 & $\mathrm{P}$ \\
\hline $\begin{array}{l}\text { Rubiaceae } \\
\text { Alibertia } \text { sp. } \\
\text { Amaioua guianensis Aubl. } \\
\text { Chimarrhis sp. } \\
\text { Duroia macrophylla } \text { Huber } \\
\text { Genipa americana L. } \\
\text { Isertia } \text { sp. }\end{array}$ & $\begin{array}{c}\text { Bostinha } \\
\text { Canela-de-veado } \\
\text { Morceguinho } \\
\text { Cabeça-de-urubu } \\
\text { Jenipaporana } \\
\text { Goiaba-de-anta }\end{array}$ & $\begin{array}{r}19 \\
1 \\
18 \\
43 \\
1 \\
1\end{array}$ & $\begin{array}{c}5,76 \\
0,30 \\
5,45 \\
13,03 \\
0,30 \\
0,30\end{array}$ & $\begin{array}{l}\mathrm{P} \\
\mathrm{Si} \\
\mathrm{Si} \\
\mathrm{Si} \\
\mathrm{P} \\
\mathrm{Si}\end{array}$ \\
\hline $\begin{array}{l}\text { Rutaceae } \\
\quad \text { Zanthoxylum pterota (L.) Kunth }\end{array}$ & Espinheiro & 1 & 0,30 & $\mathrm{P}$ \\
\hline $\begin{array}{l}\text { Salicaceae } \\
\qquad \text { Salix martiana Leyb. }\end{array}$ & Orana & 1 & 0,30 & $\mathrm{Sc}$ \\
\hline $\begin{array}{l}\text { Sapotaceae } \\
\text { Manilkara huberi (Ducke) Chevalier } \\
\text { Manilkara } \mathrm{sp} . \\
\text { Pouteria guianensis Aubl. } \\
\text { Pouteria } \text { sp. }\end{array}$ & $\begin{array}{l}\text { Maçaranduba } \\
\text { Maparajuba } \\
\text { Abiurana } \\
\text { Folhinha }\end{array}$ & $\begin{array}{r}9 \\
1 \\
236 \\
12\end{array}$ & $\begin{array}{c}2,73 \\
0,30 \\
71,52 \\
3,64\end{array}$ & $\begin{array}{l}\text { St } \\
\text { St } \\
\text { St } \\
\text { St }\end{array}$ \\
\hline $\begin{array}{l}\text { Sterculiaceae } \\
\text { Guazuma ulmifolia Lam } \\
\text { Theobroma martiana D. Dietr. } \\
\text { Theobroma subincanum Mart. }\end{array}$ & $\begin{array}{l}\text { Mutamba-verdadeira } \\
\text { Cacau-da-mata } \\
\text { Cupu-do-mato }\end{array}$ & $\begin{array}{r}6 \\
8 \\
13\end{array}$ & $\begin{array}{l}1,82 \\
2,42 \\
3,94\end{array}$ & $\begin{array}{l}\mathrm{P} \\
\mathrm{Si} \\
\mathrm{Si}\end{array}$ \\
\hline $\begin{array}{l}\text { Ulmaceae } \\
\text { Trema } \mathrm{sp} .\end{array}$ & Chumbinho & 2 & 0,61 & $\mathrm{P}$ \\
\hline $\begin{array}{l}\text { Verbenaceae } \\
\quad \text { Vitex cymosa Bertero ex Spreng. }\end{array}$ & Tarumã & 3 & 0,91 & $\mathrm{Si}$ \\
\hline $\begin{array}{l}\text { Violaceae } \\
\text { Rinorea flavescens (Aubl.) Kuntze } \\
\text { Rinorea guianensis Aubl. } \\
\text { Rinorea macrocarpa (C. Mart. ex Eichler) Kuntze }\end{array}$ & $\begin{array}{l}\text { Canela-de-jacu } \\
\text { Acariquarana } \\
\text { Canela-de-velho }\end{array}$ & $\begin{array}{r}1 \\
1 \\
23\end{array}$ & $\begin{array}{l}0,30 \\
0,30 \\
6,97\end{array}$ & $\begin{array}{l}\mathrm{Si} \\
\mathrm{Si} \\
\mathrm{Si}\end{array}$ \\
\hline $\begin{array}{l}\text { Vochysiaceae } \\
\text { Callisthene sp. } \\
\text { Vochysia ferruginea Mart. }\end{array}$ & $\begin{array}{l}\text { Vassourinha } \\
\text { Quaruba }\end{array}$ & $\begin{array}{r}13 \\
3\end{array}$ & $\begin{array}{l}3,94 \\
0,91\end{array}$ & $\begin{array}{l}\mathrm{Si} \\
\mathrm{St}\end{array}$ \\
\hline Indeterminada 1 & ------- & 1 & 0,30 & $\mathrm{Sc}$ \\
\hline Indeterminada 2 & ------- & 1 & 0,30 & $\mathrm{Sc}$ \\
\hline Indeterminada 3 & ------- & 1 & 0,30 & $\mathrm{Sc}$ \\
\hline Indeterminada 4 & ------- & 1 & 0,30 & $\mathrm{Sc}$ \\
\hline Indeterminada 5 & ------- & 1 & 0,30 & $\mathrm{Sc}$ \\
\hline Indeterminada 6 & ------ & 1 & 0,30 & $\mathrm{Sc}$ \\
\hline Indeterminada 7 & ------- & 1 & 0,30 & $\mathrm{Sc}$ \\
\hline & Total & 3.050 & 924,24 & \\
\hline
\end{tabular}

$\mathrm{N}_{\mathrm{i}}$ - Número de indivíduos da espécie i; DA - Densidade Absoluta (ind. ha ${ }^{-1}$ ); GE - Grupo ecológico; P - Pioneira; Si - Secundária inicial; St - Secundária tardia; SC - Sem caracterização. 
O sistema de amostragem adotado forneceu indicações de ser apropriado para este estudo, pois, se verificou que as 66 unidades amostrais alocadas foram suficientes para atender ao erro de amostragem admissível de $10 \%$ a 95\% de probabilidade. O erro de amostragem calculado, levando-se em consideração o número de indivíduos por parcela, foi de apenas 5,7\%.

As famílias com maior riqueza em espécies foram Caesalpiniaceae, com quatorze espécies (10,53\%), seguidas de Moraceae com dez (7,52\%), Lauraceae com nove $(6,77 \%)$, Fabaceae e Rubiaceae com seis (4,51\%), Lecythidaceae e Euphorbiaceae com cinco (3,76\%), Chrysobalanaceae, Clusiaceae, Myristicaceae e Sapotaceae com quatro (3\%), e Annonaceae, Apocynaceae, Meliaceae e Sterculiaceae, representadas por três espécies $(2,25 \%)$. Pertencem a estas famílias $62,36 \%$ das espécies identificadas. Oliveira et al. (2008), em estudo de composição e diversidade florístico-estrutural em Floresta Densa de Terra Firme na Amazônia Central, também mencionaram Caesalpiniaceae, Lauraceae, Moraceae, Fabaceae, Lecythidaceae, Euphorbiaceae, Chrysobalanaceae, Sapotaceae e Annonaceae entre familias de maior diversidade.

As famílias que apresentaram maior número de indivíduos foram Sapotaceae, Moraceae, Mimosaceae, Lecythidaceae e Chrysobalanaceae, correspondendo a $35,97 \%$ dos indivíduos amostrados na área. Esses resultados corroboram parcialmente os trabalhos de Oliveira \& Amaral (2004) em uma Floresta de Vertente na Amazônia Central, Lima Filho et al. (2004) em uma Floresta de Terra Firme em Cachoeira Portela, PA, Oliveira \& Amaral (2005) em um sub-bosque de Terra Firme na Amazônia Central e Pinheiro et al. (2007) em área de preservação permanente no leste da Amazônia, que apontaram o predomínio das famílias Sapotaceae, Lecythidaceae, Burseraceae e Chrysobalanaceae.

Segundo Parthasarathy (1999), é característico, em muitas florestas, um pequeno número de espécies com alta densidade e um grande número de espécies com baixa densidade. Essa característica também foi observada neste estudo, onde um total de 65 (49\%) das 133 espécies amostradas apresentou densidade inferior a seis indivíduos e 37 espécies (28\%) apresentaram apenas um indivíduo amostrado. Esses resultados corroboram parcialmente com Oliveira \& Amaral (2004), Oliveira \& Amaral (2005) e Silva et al. (2008), ao estudarem áreas de Floresta Ombrófila Densa em Manaus, AM, onde registraram que $40 \%$ a $60 \%$ das espécies levantadas foram representadas por apenas um indivíduo.

$\mathrm{Na}$ área estudada, algumas espécies como Tabebuia serratifolia, Cordia sp., Cochlospermum orinoccense, Byrsonima sp. e Minquartia guianensis foram representadas por apenas um indivíduo, sendo consideradas "localmente raras" (Oliveira et al., 2008). De acordo com Silva (2006), as espécies raras são restritas a um conjunto de fatores ambientais que as mantêm, assim como limitações na dispersão, de modo que as mesmas estão sujeitas a substituição no ambiente estudado, ou seja, suscetíveis à extinção no local.

As espécies que se destacaram com o maior número de indivíduos amostrados foram: Eschweilera odora, Pouteria guianensis, Inga sp., Licania oblongifolia, Maquira guianensis, Xylopia nitida, Eugenia paraensis, Virola multiflora, Protium heptaphyllum e Licania canescens (Figura 2). Juntas, essas dez espécies representam $57,5 \%$ do total de indivíduos amostrados.

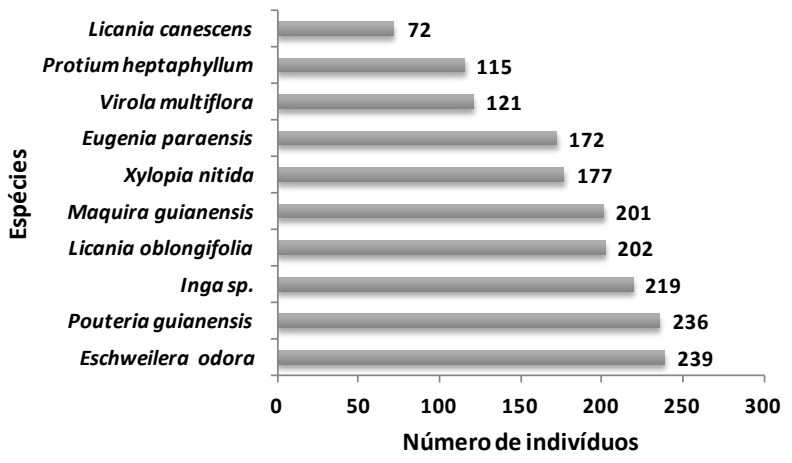

Figura 2. Espécies com maior representatividade em número de indivíduos, em uma área de Floresta Ombrófila Densa de Terras Baixas, na RDS Uacari, Carauari, AM.

Comparando o número de indivíduos com outros estudos florísticos na região leste e nordeste do Pará, como os de Pinheiro et al. (2007) e Trindade et al. (2007), que usaram um nível de inclusão superior, porém, amostraram uma área em média cinco vezes maior que a área amostrada nesse estudo, verificou-se que a área do presente estudo possui maior número de indivíduos por hectare. Esse número pode estar relacionado ao fato do estudo ter sido desenvolvido na área de uma reserva onde as comunidades só utilizam madeira para uso próprio, e as espécies as quais têm utilidades não madeireiras, como Andiroba (Carapa guianensis), Copaíba (Copaifera sp.), Castenheira (Bertholletia 
excelsa) e a seringueira (Hevea brasiliensis), não são cortadas, pois, além de serem espécies protegidas por Lei, os ribeirinhos comercializam suas sementes e látex.

Lyons et al. (2005) afirmam que o conjunto das espécies pouco abundantes pode ter uma importância ecológica e ser responsável por diversas funções no ecossistema, como aumentar a resistência da comunidade contra invasores e retenção de nutrientes.

Quanto à classificação sucessional, constatou-se que 21 espécies compõem o grupo das pioneiras (16\%), 46 secundárias iniciais (34\%), 54 secundárias tardias (41\%) e 12 sem caracterização (9\%).

Ainda que tenha sido verificada uma predominância das espécies secundárias tardias, foram as espécies de início de sucessão (pioneiras + secundárias iniciais) que compuseram a maioria da flora encontrada (50\%). De acordo com Gandolfi et al. (1995), o grande número de espécies iniciais sugere a predominância de uma condição jovem.
A elevada densidade de espécies pertencentes ao grupo das tardias pode estar relacionada ao fato do levantamento ter sido realizado em uma área com histórico de perturbação, pois aproximadamente $69 \%$ das espécies secundárias tardias encontradas no levantamento eram exploradas comercialmente, como por exemplo: Carapa guianensis, Couratari oblongifolia, Apuleia molaris, Tabebuia serratifolia, Mezilaurus itauba, Manilkara huberi e Hevea brasiliensis. Porém, anos antes da criação da Reserva de Desenvolvimento Sustentável Uacari (2005), essa atividade não mais aconteceu e com isso pode-se observar que o atual estado da reserva tem favorecido o estabelecimento de espécies secundárias tardias, o que demonstra que a conservação está sendo eficiente.

Ao analisar a estrutura horizontal por grupo ecológico, observa-se que em todos os parâmetros fitossociológicos os grupos das secundárias iniciais e tardias se destacaram (Tabela 2).

Tabela 2: Valores dos parâmetros da estrutura horizontal por grupo ecológico, das espécies amostradas em uma área de Floresta Ombrófila Densa de Terras Baixas, na RDS Uacari, Carauari, AM.

\begin{tabular}{crrrrrrrr}
\hline Grupos ecológicos & \multicolumn{1}{c}{$\mathbf{N}_{\mathbf{i}}$} & \multicolumn{1}{c}{ DA } & \multicolumn{1}{c}{ DR } & FA & FR & DoA & DoR & VI \\
\hline Pioneiras - P & 290 & 87,88 & 9,41 & 284,85 & 12,75 & 3,070 & 8,583 & 30,84 \\
Secundárias iniciais - Si & 1.777 & 538,48 & 58,46 & $1.072,73$ & 48,00 & 16,327 & 45,647 & 151,91 \\
Secundárias tardias - St & 938 & 284,24 & 30,65 & 825,76 & 36,95 & 15,480 & 43,278 & 110,98 \\
Sem caracterização - Sc & 45 & 13,64 & 1,48 & 51,52 & 2,30 & 0,891 & 2,492 & 6,27 \\
\hline Total & 3.050 & 924,24 & 100 & $2.234,86$ & 100 & 35,768 & 100 & 300 \\
\hline
\end{tabular}

$\mathrm{N}_{\mathrm{i}}$ - Número de indivíduos da espécie i; DA - densidade absoluta (ind. ha $\left.{ }^{-1}\right)$; DR - densidade relativa (\%); FA - frequência absoluta (\%); FR - frequência felativa (\%); DoA - dominância absoluta $\left(\mathrm{m}^{2} \mathrm{ha}^{-1}\right)$; DoR - dominância relativa (\%) e VI - valor de importância.

Os dados sobre o número de indivíduos por grupo ecológico mostram que os indivíduos de estágios finais de sucessão estão encontrando condições mais favoráveis para o seu desenvolvimento. Dentre as espécies pioneiras encontradas no trecho de Floresta Ombrófila Densa de Terras Baixas estudado, as mais abundantes foram: Pourouma sp. (56), Neea oppositifolia (52), Ormosia sp. (41), Abarema sp. (29) e Alibertia sp. (19) que juntas corresponderam a $67,93 \%$ dos indivíduos amostrados pertencentes a este grupo ecológico. A frequência indicou que os indivíduos das espécies classificadas como secundárias iniciais e secundárias tardias estão bem distribuídos sobre a área amostrada.
O valor de importância permite uma visão mais ampla da posição da espécie, caracterizando sua importância na população em estudo (Odum, 1983). Dentre as dez espécies de maior VI, oito se encontram no grupo das secundárias iniciais (Eschweilera odora, Licania oblongifolia, Inga sp., Xylopia nitida, Maquira guianensis, Eugenia paraensis, Protium heptaphyllum e Licania canescens) e duas no grupo das secundárias tardias (Pouteria guianensis e Virola multiflora).

A distribuição diamétrica das árvores encontradas na área de estudo apresentou padrão característico para florestas tropicais inequiâneas, com a distribuição em forma de "J" invertido (Souza et al., 2006), ou seja, maior presença de indivíduos nas menores classes diamétricas (Figura 3). 


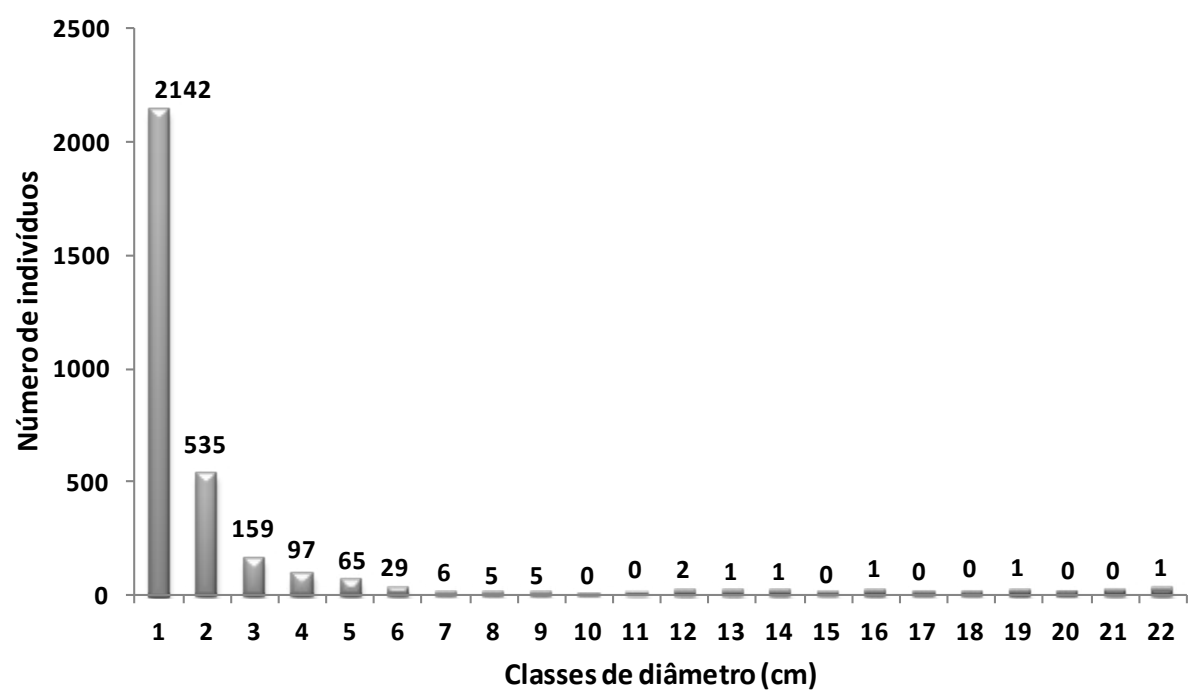

Figura 3. Distribuição dos indivíduos por classe de diâmetro, em uma área de Floresta Ombrófila Densa de Terras Baixas, na RDS Uacari, Carauari, AM.

Ao analisar o gráfico da distribuição diamétrica, fica evidenciado que $70,23 \%$ dos indivíduos amostrados se concentram na primeira classe de diâmetro que vai de $7,95 \mathrm{~cm}$ a $17,95 \mathrm{~cm}$, evidenciando a alta densidade de indivíduos jovens. As duas classes seguintes reúnem apenas $22,75 \%$ do total de indivíduos amostrados, percebendo-se uma nítida redução entre o número de indivíduos da primeira classe para a segunda e da segunda para a terceira. Essas três primeiras classes reúnem aproximadamente $93 \%$ do número total de indivíduos amostrados.

A grande disparidade entre o número de indivíduos na primeira e na segunda classe comprova a ocorrência de uma floresta secundária, evidenciando que a área em estudo já sofreu uma forte ação antrópica. Antes da criação da reserva, os próprios moradores extraiam madeiras e vendiam de forma ilegal aos madeireiros da região, porém, atualmente, essa atividade não é mais praticada na RDS Uacari, dando lugar à extração do látex para fabricação da borracha, dos óleos vegetais, mel, cipós e o artesanato, garantindo com isso a sustentabilidade.

Não foi observada a presença de indivíduos nas classes $10,11,15,17,18,20$ e 21 . Já nas classes 13, $14,16,19$ e 22 , as espécies aparecem apenas com um indivíduo, dentre essas, Licania oblongifolia (secundária inicial), Xylopia nitida (secundária inicial) e Apuleia molaris (secundária tardia) estão bem representadas nas primeiras classes. As espécies Couepia guianensis (secundária tardia) e Copaifera multijuga (secundária tardia), também representadas por apenas um indivíduo, não apresentaram distribuição uniforme, estando ausente nas primeiras classes de diâmetro.

\section{Conclusões}

A análise do número de espécies nos diferentes grupos ecológicos indica que o trecho de Floresta Ombrófila Densa de Terras Baixas localizado na Reserva de Desenvolvimento Sustentável Uacari (RDS - Uacari) no Município de Carauari, Amazonas, apresenta características de uma floresta em estágio inicial no desenvolvimento sucessional, pois houve o predomínio de espécies de início de sucessão (pioneiras + secundárias iniciais).

A distribuição diamétrica apresentou padrão característico para florestas tropicais inequiâneas, com distribuição em forma de "J" invertido. A grande diferença entre o número de indivíduos na primeira e na segunda classe comprova a ocorrência de uma floresta secundária.

\section{Agradecimentos}

À Coordenação de Aperfeiçoamento de Pessoal de Nível Superior (Capes), pela concessão de bolsa, ao Centro Estadual de Unidades de Conservação (Ceuc), pelo apoio logístico e financeiro, e aos comunitários da RDS Uacari, pelo acolhimento e ajuda em campo. 


\section{Referências}

AMARAL, D. D. do.; VIEIRA, I. C. G.; ALMEIDA, S. S. de.; SALOMÃO, R. de P.; SILVA, A. S. L. da.; JARDIM, M. A. G. Checklist da flora arbórea de remanescentes florestais da região metropolitana de Belém e valor histórico dos fragmentos, Pará, Brasil. Boletim do Museu Paraense Emílio Goeldi, Belém, PA, v.4, n. 3, p. 231-289, 2009.

ANDRETTI, C. B.; COSTA, T. V. V.; COHN-HALT, M. Diagnóstico sócio-agroextrativista e ambiental da Reserva de Desenvolvimento Sustentável Uacari. Carauari: Secretaria de Projetos Especiais/SDS, 2006. 246 p. Relatório técnico.

BRASIL. Ministério das Minas e Energia. Departamento Nacional da Produção Mineral. Projeto RADAMBRASIL. Folha SB. 19 Juruá: geologia, geomorfologia, pedologia, vegetação, uso potencial da terra. Rio de Janeiro, 1977. 555 p. (Levantamento de recursos naturais, v. 15).

CARVALHO, F. A.; NASCIMENTO, M. T.; BRAGA, J. M. A. Estrutura e composição florística do estrato arbóreo de um remanescente de mata atlântica submontana no município de Rio Bonito, RJ, Brasil (Mata Rio Vermelho). Revista Árvore, Viçosa, MG, v. 31, n. 4, p. 717-730, 2007.

CRONQUIST, A. The evolution and classification of flowering plants. New York: The New York Botanical Garden, 1988. 555 p.

FERRAZ, I. D. K.; LEAL FILHO, N.; IMAKAWA, A. M.; VARELA, V. P.; PIÑA-RODRIGUES, F. C. M. Características básicas para um agrupamento ecológico preliminar de espécies madeireiras da floresta de terra firme da Amazônia Central. Acta Amazônica, Manaus, v. 34, n. 4, p. 621-633, 2004.

FIGUEIREDO, P. S. de.; RIBEIRO, E. K. M. D.; LACERDA, D. M. A.; GIRNOS, E. C. Estratégia reprodutiva de Cochlospermum orinocense (Kunth) Steud.: fenologia, biologia floral e sistema de cruzamento em uma espécie pioneira de florestas na Amazônia. Revista Brasileira de Botânica, São Paulo, v. 32, n. 4, p. 781-792, 2009.

GAMA, J. R. V.; BOTELHO, S. A.; BENTES-GAMA, M. M. Composição florística e estrutura da regeneração natural de floresta secundária de várzea baixa no estuário amazônico. Revista Árvore, Viçosa, MG, v. 26, n. 5, p. 559-566, 2002.

GANDOLFI, S.; LEITÃO FILHO, H. F.; BEZERRA, C. L. E. Levantamento florístico e caráter sucessional das espécies arbustivo arbóreas de uma floresta mesófila semidecídua no município de Guarulhos, SP. Revista Brasileira de Biologia, Rio de Janeiro, v. 55, n. 4, p. 753-767, 1995.

IBAMA. Reserva Extrativista do Médio Juruá - AM: plano de utilização. Brasília, DF, [200?]. Disponível em: <http://www. ibama.gov.br/resex/mjurua/plano.htm>. Acesso em: 06 jul. 2011.

IBGE. Manual técnico da vegetação brasileira. Rio de Janeiro: CDDI-IBGE, 1992. 92 p. (Série manuais técnicos em geociências, n. 1).

LIMA FILHO, D. de A.; REVILLA, J.; AMARAL, I. L. do.; MATOS, F. D. de A.; COELHO, L. de S.; RAMOS, J. F.; SILVA, G. B. da.; GUEDES, J. de O. Aspectos florísticos de 13 hectares da área de Cachoeira Portela - PA. Acta Amazônica, Manaus, v. 34, n. 3, p. 415-423, 2004.
LYONS, K. G.; BRIGHAM, C. A.; TRAUT, B. H.; SCHWARTZ, M. W. Rare species and ecosystem functioning. Conservation Biology, California, Davis, v. 19, n. 4, p. 1019-1024, 2005.

MACIEL, M. de N. M.; WATZLAWICK, L. F.; SCHOENINGER, E. R.; YAMAJI, F. M. Efeito da radiação solar na dinâmica de uma floresta. Revista Ciências Exatas e Naturais, Guarapuava, PR, v. 4, n. 1, p. 101-114, 2002.

MARANGON, L. C.; SOARES, J. J.; FELICIANO, A. L.; BRANDÃO, C. F. L. e S. Estrutura fitossociológica e classificação sucessional do componente arbóreo de um fragmento de floresta estacional semidecidual, no município de Viçosa, Minas Gerais. Cerne, Lavras, v. 13, n. 2, p. 208-221, 2007.

MEUNIER, I. M. J.; SILVA, J. A. A.; FERREIRA, R. L. C. Inventário florestal: programas de estudo. Recife: Imprensa Universitária da UFRPE, 2002. 189 p.

MÜLLER DOMBOIS, D.; ELLEMBERG, H. Aims and methods for vegetation ecology. New York: John Wiley \& Sons, 1974. 547 p.

ODUM, E. P. Ecologia. Rio de Janeiro: Guanabara, 1983. 434 p.

OLIVEIRA, A. N. de.; AMARAL, I. L. do.; RAMOS, M. B. P.; NOBRE, A. D.; COUTO, L. B.; SAHDO, R. M. Composição e diversidade florístico-estrutural de um hectare de floresta densa de terra firme na Amazônia Central, Amazonas, Brasil. Acta Amazônica, Manaus, v. 38, n. 4, p. 627-642, 2008.

OLIVEIRA, A. N. de.; AMARAL, I. L. Aspectos florísticos, fitossociológicos e ecológicos de um sub-bosque de terra firme na Amazônia central, Amazonas, Brasil. Acta Amazônica, Manaus, v. 35, n. 1, p. 1-16, 2005.

OLIVEIRA, A. N. de.; AMARAL, I. L. Florística e fitossociologia de uma floresta de Vertente na Amazônia Central, Amazonas, Brasil. Acta Amazônica, Manaus, v. 34, n. 1, p. 21-34, 2004.

PARTHASARATHY, N. Tree diversity and distribution in undisturbed and human-impacted sites of tropical wet evergreen forest in southern Western Ghats, India. Biodiversity and Conservation, London, v. 8, n. 4, p. 1365-1381, 1999.

PAULA, A. de.; SILVA, A. F. da.; MARCO JÚNIOR, P. de; SANTOS, F. A. M. de.; SOUZA, A. L de. Sucessão ecológica da vegetação arbórea em uma Floresta Estacional Semidecidual, Viçosa, MG, Brasil. Acta Botânica Brasílica, São Paulo, v. 18, n. 3, p. 407-423, 2004.

PINHEIRO, K. A. O.; CARVALHO, J. O. P. de; QUANZ, B.; FRANCEZ, L. M. de B.; SCHWARTZ, G. Fitossociologia de uma área de preservação permanente no leste da amazônia: indicação de espécies para recuperação de áreas alteradas. Floresta, Curitiba, v. 37, n. 2, p. 175-187, 2007.

PRADO JÚNIOR, J. A. do; VALE, V. S. do; OLIVEIRA, A. P. de; GUSSON, A. E.; DIAS NETO, O. C.; LOPES, S. de F.; SCHIAVINI, I. Estrutura da comunidade arbórea em um fragmento de floresta estacional semidecidual localizada na reserva legal da fazenda Irara, Uberlândia, MG. Bioscience Journal, Uberlândia, v. 26, n. 4, p. 638-647, 2010. 
ROCHA, K. D. da; BRANDÃO, C. F. L. S.; SILVA, J. T. da; SILVA, M. A. V. da; JÚNIOR, F. T. A.; MARANGON, L. C. Classificação sucessional e estrutura fitossociológica do componente arbóreo de um fragmento de mata atlântica em Recife, Pernambuco, Brasil. Magistra, Cruz das Almas, v. 20, n. 1, p. 46-55, 2008.

RODRIGUES, R.; GANDOLFI, S.; DIAS, L.; MELLO, J. Restauração de florestas tropicais: subsídios para uma definição metodológica e indicadores de avaliação e monitoramento. In: DIAS, L. E.; MELLO. J. W. (Eds.). Recuperação de áreas degradadas. Viçosa, MG: UFV; Sociedade Brasileira de Recuperação de Áreas Degradadas, 1998. p. 203-215.

SANTOS, J. H. da S.; FERREIRA, R. L. C.; SILVA, J. A. A. da; SOUZA, A. L. de; SANTOS, E. de S.; MEUNIER, I. M. J. Distinção de grupos ecológicos de espécies florestais por meio de técnicas multivariadas. Revista Árvore, Viçosa, MG, v. 28, n. 3 , p. 387-396, 2004.

SANTOS, H. G. dos; JACOMINE, P. K. T.; ANJOS, L. H. C. dos; OLIVEIRA, V. A. de; OLIVEIRA, J. B. de; COELHO, M. R.; LUMBRERAS, J. F.; CUNHA, T. J. F. da. Sistema brasileiro de classificação de solos. 2. ed. Brasília, DF: Embrapa Informação Tecnológica; Rio de Janeiro: Embrapa Solos, 2006. 412 p.

SILVA, A. F.; OLIVEIRA, R. V.; SANTOS, N. R. L.; DE PAULA, A. Composição florística e grupos ecológicos das espécies de um trecho de floresta semidecidua submontana da Fazenda São Geraldo, Viscosa, Minas Gerais. Revista Árvore, Viçosa, MG, v. 27, n. 3, p. 311-319, 2003.

SILVA, K. E.; MATOS, F. D. A.; FERREIRA, M. M. Composição florística e fitossociológica de espécies arbóreas do Parque Fenológico da Embrapa Amazônia Ocidental. Acta Amazônica, Manaus, v. 38, n. 2, p. 213-222, 2008.
SILVA, S. M. G. Descritores fitossociológico: estruturais para elaboração de diretrizes técnicas visando a conservação in situ da diversidade florestal na fazenda experimental da UFAM. 2006. 132 f. Dissertação (Mestrado em Ciências Florestais e Ambientais) - Universidade Federal do Amazonas, Manaus.

SOARES, C. P. B.; PAULA NETO, F.; SOUZA, A. L. Dendrometria e inventário florestal. Viçosa, MG: UFV, 2007. 276 p.

SOUZA, D. R. de.; SOUZA, A. L. de; LEITE, H. G.; YARED, J. A. G. Análise estrutural em floresta ombrófila densa de terra firme não explorada, Amazônia Oriental. Revista Árvore, Viçosa, MG, v. 30, n. 1, p. 75-87, 2006.

SOUZA, P. B. Diversidade florística e atributos pedológicos ao longo de uma encosta com floresta estacional semidecidual submontana, zona de amortecimento do Parque Estadual do Rio Doce, MG. 2008. 138 f. Tese (Doutorado em Botânica) Universidade Federal de Viçosa, Viçosa, MG.

SWAINE, M. D.; WHITMORE, T. C. On the definition of ecological species groups in tropical rain forests. Vegetatio, Amsterdam, v. 75, p. 81-86, 1988.

TRINDADE, M. J. S.; ANDRADE, C. R.; SOUSA, L. A. S. Florística e fitossociologia da reserva do Utinga, Belém, Pará, Brasil. Revista Brasileira de Biociências, Porto Alegre, v. 5, p. 234-236, 2007. (Suplemento, n. 2).

VIANELLO, R. L.; ALVES, A. R. Meteorologia básica e aplicações. Viçosa, MG: UFV, 2000. 449 p. 